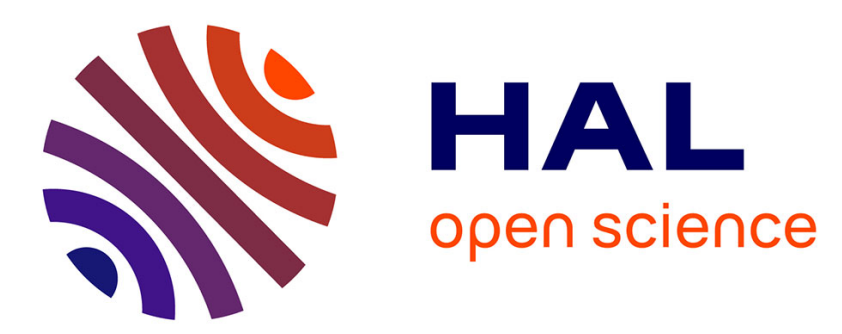

\title{
Short-Sighted Robust LPV Model Predictive Control: Application to Semi-Active Suspension Systems
}

\author{
Marcelo Menezes Morato, Julio E Normey-Rico, Olivier Sename
}

\section{To cite this version:}

Marcelo Menezes Morato, Julio E Normey-Rico, Olivier Sename. Short-Sighted Robust LPV Model Predictive Control: Application to Semi-Active Suspension Systems. ECC 2021 - 20th European Control Conference, Jun 2021, Rotterdam (on line), Netherlands. 10.23919/ECC54610.2021.9655162 . hal-03170815

\section{HAL Id: hal-03170815 https://hal.science/hal-03170815}

Submitted on 16 Mar 2021

HAL is a multi-disciplinary open access archive for the deposit and dissemination of scientific research documents, whether they are published or not. The documents may come from teaching and research institutions in France or abroad, or from public or private research centers.
L'archive ouverte pluridisciplinaire HAL, est destinée au dépôt et à la diffusion de documents scientifiques de niveau recherche, publiés ou non, émanant des établissements d'enseignement et de recherche français ou étrangers, des laboratoires publics ou privés. 


\title{
Short-Sighted Robust LPV Model Predictive Control: Application to Semi-Active Suspension Systems
}

\author{
Marcelo M. Morato ${ }^{1,2}$, Julio E. Normey-Rico ${ }^{1}$ and Olivier Sename ${ }^{2}$
}

\begin{abstract}
This paper develops a novel Linear Parameter Varying (LPV) Model Predictive Control (MPC) algorithm for Semi-Active Suspension systems. The current state-of-theart comprises two possible implementations: a) to consider the future variations of the LPV scheduling variables as uncertainties, thereby solving a robust optimization, which is usually time-consuming; or b) to estimate the future scheduling variables and solve a sub-optimal quadratic program, which can be evaluated rapidly. This paper proposes a control paradigm in between these paths, considering a robust min-max procedure with small predictions horizons, being implementable within the short $5 \mathrm{~ms}$ sampling period of the suspension system. The method includes terminal ingredients, derived via LMIs, that ensure input-to-state stability and recursive feasibility. Realistic simulations show the effectiveness of the proposed method, when compared against a nonlinear MPC and a sub-optimal LPV MPC. The results show that the method is indeed able to run in real-time (in the order of milliseconds), almost as fast as the sub-optimal MPC, while still guaranteeing good safety and comfort performances for the vehicle.
\end{abstract}

\section{INTRODUCTION}

Automotive suspension systems provide better safety and passenger comfort. Semi-Active (SA) suspensions are standard in many high-range cars and topic of a good deal of academic and industrial research [1]. The major investigation problem is how to provide real-time laws for the controllable damper, which operates within some milliseconds, ensuring performance requirements, such as more comfortable rides altogether with easier maneuvering, while accounting for the SA (input) dissipativity constraints. Recently, some papers have demonstrated the application of Model Predictive Control (MPC) to this problem [2]-[4], which is a topic increasingly sought by the automotive industry, in particular when considering more complex, nonlinear models for the vehicle dynamics. MPC is indeed a good option to address the issue of SA suspension control, since it is able to elegantly deal with the SA input and state constraints [5].

In this paper, we are concerned with quasi-/Linear Parameter Varying (qLPV/LPV) model structures, which are able to $\mathrm{embed}^{1} / \mathrm{represent}$ the complex, nonlinear vertical dynamics of a SA suspension system [7]. A recent survey

The Authors thank CNPq and ITEA3 for supporting projects 304032/2019-0 and 15016 EMPYSHIS, respectively.

${ }^{1}$ Renewable Energy Research Group (GPER), Universidade Federal de Santa Catarina (UFSC), Florianópolis, Brazil. e-mail: marcelomnzm@gmail.com; ${ }^{2}$ Univ. Grenoble-Alpes, CNRS, Grenoble INP ${ }^{\top}$, GIPSA-Lab,38000 Grenoble, France. $T$ Institute of Engineering, Univ. Grenoble-Alpes.

${ }^{1}$ Many nonlinear processes can indeed be represented within qLPV model structures, as long as the Linear Differential Inclusion (LDI) property is respected [6]; in this case, the nonlinearities are appropriately "hidden" to the LPV scheduling parameters. details the possibilities of issuing LPV MPC by exploiting the linearity properties of the input/output channels [8]; the main difficulty is how to cope with the future scheduling parameters, which are unknown. Regarding this topic, the state-of-the-art comprises, basically, two main threads [8]: robust "min-max" algorithms and sub-optimal approaches.

On one hand, robust LPV MPC methods [9] ensure that the performances hold for the worst-case scenario given w.r.t. the LPV parameters $\rho$. The major drawback is that the max. step is computationally costly, which forbids real-time applications. On the other hand, literature displays a variety of sub-optimal methods that replace the Nonlinear Problem (NP) by a Quadratic Problem (QP) based on fixed/adaptative prediction guesses for $\rho$ [10], [11]. Their major drawback is that, by being sub-optimal, local minima can be found, which might not ensure sufficient performances.

The designer faces a complexity vs. optimality trade-off in order to synthesize MPC for systems with LPV models [8]: One can either opt for ensuring robust stabilization of the process with performances guarantees, with numerically tougher and more time-consuming implementation, or use sub-optimal QPs, which are able to operate in real-time but may result in poor performances. Recent SA suspension literature shows only sub-optimal LPV MPC approaches [3], [4], since robust procedures are seemingly unable to operate within their short sampling periods. Methods in between these two lanes are missing, which is a literature gap.

\section{A. Problem Statement}

Motivated by this lacuna, the goal of this paper is to propose an alternative, robust MPC design method for SA suspensions systems with LPV models. The method is hereafter named as "short-sighted". Its innovation is not the LPV MPC problem itself, but rather the heuristically-tuned shortsighted paradigm, which is a novel approach to the theme.

The main contribution of this short-sighted paradigm consists in solving the LPV MPC problem with short prediction horizons, of only some discrete-time steps ahead, which enables the operation of the proposed method for the realtime SA suspensions application. The MPC is formulated as a robust min-max procedure, comprising the use of parameter-dependent terminal ingredients. An input-to-state stability proof is accordingly provided.

The considered problem is how to design a robust LPV MPC algorithm for SA suspension systems which is able to balance the (road holding, passenger comfort) performances vs. complexity trade-off, respecting the sampling period threshold limitation. Furthermore, despite using small 
horizons, the regulation performance should be maintained, which means that input-to-state stability and recursive feasibility properties are required.

The paper is organized as follows: Sec. II details the shortsighted LPV MPC problem setup, discussing the prediction problem with respect to the scheduling variable. The proposed short-sighted heuristic to tune the prediction horizon is proposed therein. Sec. III details the offline computation of the optimization terminal ingredients, which comprises an LMI-solvable remedy that ensures recursive feasibility and input-to-state stability of the closed-loop system. Sec. IV presents the application of the method to the nonlinear SA suspension problem and comparisons to other methods. General conclusions are drawn in Sec. V.

\section{Short-Sighted LPV MPC Algorithm SetuP}

\section{A. The LPV Process Model}

In this paper, we consider ${ }^{2}$ the following LPV model:

$$
\begin{aligned}
x(k+1) & =A(\rho(k)) x(k)+B(\rho(k)) u(k) \\
& +B_{2}(\rho(k)) w(k), \\
\rho(k) & =\rho(k-1)+\partial \rho(k) \in \mathcal{P},
\end{aligned}
$$

where $x: \mathbb{N} \rightarrow \mathbb{R}^{n_{x}}$ stands for the system states, $u$ : $\mathbb{N} \rightarrow \mathbb{R}^{n_{u}}$ represents the control inputs and $w: \mathbb{N} \rightarrow$ $\mathbb{R}^{n_{w}}$ are unmeasured load disturbances. Note that $\rho(k)$ : $\mathbb{N} \rightarrow \mathbb{R}^{n_{p}}$ denotes a bounded scheduling variable, which is known, online, at each instant $k$, but generally unknown for any future instant $k+j, \forall j \in \mathbb{N}^{+}$.

This process must be regulated in such way that the state trajectories are steered to the origin, in an admissible manner, despite disturbances. The admissible realization follows:

Assumption 1: The admissible operation for the states is given by a 2-norm upper bound on each entry $x_{j}$, this is:

$$
\mathcal{X}:=\left\{x \in \mathbb{R}^{n_{x}} \mid\left\|x_{j}\right\|^{2} \leq \bar{x}_{j}, \forall j \in \mathbb{N}_{\left[1, n_{x}\right]}\right\} \text {. (3) }
$$

Assumption 2: The admissible control inputs are those within the following set:

$$
\mathcal{U}:=\left\{u \in \mathbb{R}^{n_{u}} \mid\left\|u_{j}\right\|^{2} \leq \bar{u}_{j}, \forall j \in \mathbb{N}_{\left[1, n_{u}\right]}\right\} \text {. (4) }
$$

Assumption 3: The scheduling variable evolves with bounded rates of variation, i.e. $\partial \rho \in \partial \mathcal{P}$. We note that this is a very reasonable hypothesis for any real application ${ }^{3}$.

Assumption 4: The states are measurable for all sampling instants and the process is controllable. This means that the control policy can be formulated under state-feedback: $u(k)=K(k) x(k)$.

\section{B. Predictive Control Framework}

In order to ensure this regulation goal, we consider a predictive control algorithm. We proceed by detailing how MPC can be applied within a short-sighted paradigm. The essential idea behind MPC is to use a finite-horizon cost, which embeds the performance objectives of the system

\footnotetext{
${ }^{2}$ Notation $\nu(k+i \mid k)$ denotes the prediction of $\nu$ at instant $k+i$, computed based on the information available at instant $k . \mathbb{N}_{[a, b]}$ denotes the set of natural numbers from $a$ to $b$.

${ }^{3}$ Assuming that $\rho$ varies arbitrary implies in conservative synthesis.
}

within a prediction horizon. The implementation resides in minimizing this cost w.r.t. a control signal sequence $U_{k}$, using the model of the system to make predictions for the future variables, along the horizon. The optimization also includes the operational constraints of the process variables (admissibility region, given by Assumptions 1 and 2). Generically, we consider the following performance cost:

$$
\begin{aligned}
J(x, u, k) & =\overbrace{\sum_{i=1}^{N_{p}}\|x(k+i \mid k)\|_{Q}^{2}+\|u(k+i-1 \mid k)\|_{R}^{2}}^{\ell(\cdot)} \\
& +V\left(x\left(k+N_{p} \mid k\right)\right)
\end{aligned}
$$

where $Q$ and $R$ are positive weighting matrices and $N_{p} \geq 2$ is the prediction horizon. The terminal cost $V\left(x\left(k+N_{p} \mid k\right)\right)>0$ is used to ensure input-to-state stability and recursive feasibility.

MPC considers a moving-window strategy. Therefore, at each sampling instant $k$, since $x(k)$ and $\rho(k)$ are known, an optimization problem is solved, whose solution is $U_{k}=$ $\left[u(k \mid k) \quad \ldots u\left(k+N_{p}-1 \mid k\right)\right] \in \mathbb{R}^{n_{u} \times N_{p}}$. The first input of this vector $u(k \mid k)=I_{1} U_{k}$ is applied to the process. The horizon slides forward and the procedure is updated.

Since $\partial \rho(k+j)$ is an unknown variable along the future horizon (i.e. for all $j \in \mathbb{N}^{+}$), this variable is considered as an uncertainty, from the control viewpoint. The corresponding robust control optimization resides in minimizing the worst-case/maximal performance cost $\bar{J}(x, u, k)=$ $\max _{\partial \rho(k+j) \in \partial \mathcal{P}} J(x, u, k)$. The resulting min-max optimization comprises the operation of a maximization NP followed by a minimization QP:

$$
\begin{aligned}
U_{k}^{\star}= & \arg \min _{U_{k}}\left(\max _{\partial \rho(k+j) \in \partial \mathcal{P}} \ell(x, u, k)+V(\cdot)\right) \\
\text { s.t. } \quad & \overbrace{x(k+i+1 \mid k)=A(\rho(k+i)) x(k+i \mid k)}^{\text {LPV Process Model }} \\
& +B(\rho(k+i)) u(k+i \mid k), \forall i \in \mathbb{N}_{\left[1, N_{p}\right]}, \\
& \overbrace{u(k+i-1 \mid k) \in \mathcal{U}}^{\text {Control Input Admissibility }}, \forall i \in \mathbb{N}_{\left[1, N_{p}\right]}, \\
& \overbrace{x(k+i \mid k) \in \mathcal{X}}^{\text {Admissible Process Operation }}, \forall i \in \mathbb{N}_{\left[1, N_{p}\right]}, \\
& \overbrace{x\left(k+N_{p} \mid k\right) \in \mathbf{X}_{f}}^{\text {Terminal Set Constraint }},
\end{aligned}
$$

where $\mathbf{X}_{f}$ and $V(\cdot)$ are the terminal ingredients. This constrained optimization ensures that the regulation goal is achieved with an admissible operation, respecting Assumptions 1 and 2.

\section{Short-Sighted Heuristic}

A complexity barrier is included to this min-max procedure, in such way that it can be applied in real-time; the prediction horizon $N_{p}$ is chosen according to the proposed short-sighted heuristic:

$$
N_{p}=\left\{\arg \min _{N_{p}}\{J \text { in Eq. (6) }\} \mid t_{c}<T_{s}\right\},
$$


being $N_{p}$ the maximal horizon size, $t_{c}$ the average computational time needed by the embedded solver to analytically evaluate Eq. (6) and $T_{s}$ the sampling period of the discretetime process in Eq. (1). The horizon size $N_{p}$ will depend on the processor, on the used solver and on the system size. We note that, for the majority of time-critical processes (for which $T_{s}$ is given in the millisecond range), $N_{p}$ is simply a few steps.

Despite using a small horizon size, terminal ingredients are used to ensure input-to-state stability and recursive feasibility of the MPC algorithm. The drawback of such short-sighted paradigm is that the performances may be deteriorated w.r.t. those obtained with a larger $N_{p}$ or with an NMPC approach. We recall that the execution of an NMPC is computationally unattractive because of its general nonlinear dependence of the predicted states on the future control inputs and states [12], i.e. "full-blown" NP.

Remark 1: With regard to this short-sighted paradigm, we must comment that the pitfall of performance degradation is not verified in the considered SA suspension application, as shown in Sec. IV. From an application perspective, therefore, the paradigm provides elegancy and implementation simplicity.

\section{InPUT-TO-STATE STABility PRoOF}

In this Section, we offer a Theorem to construct the LPV MPC terminal ingredients and demonstrate the input-to-state stability and recursive feasibility properties of the proposed algorithm. The following procedures are independent of the short-sighted heuristic as long as $N_{p}>2$. The usual approach with terminal ingredients [13] resides in ensuring that closed-loop energy-dissipating conditions are met by (a) the terminal set $\mathbf{X}_{f}$ and by (b) the terminal cost $V(\cdot)$, computed w.r.t. a nominal ${ }^{4}$ state-feedback controller $u(k)=$ $K(\cdot) x(k)$.

In this paper, we consider that there exists a parameterdependent state-feedback gain $K: \mathbb{R}^{n_{p}} \rightarrow \mathbb{R}^{n_{x} \times n_{u}}$. We consider an ellipsoidal terminal set:

$$
\mathbf{X}_{f}(\rho)=\left\{x \mid x^{T} P(\rho) x \leq \alpha_{P}\right\},
$$

which is centered at the origin and has a radius of $\alpha_{P}$. Furthermore, this terminal set is a sub-level set of terminal cost $V(\cdot)$, taken as a Lyapunov function:

$$
V(x, \rho)=x^{T} P(\rho) x .
$$

Theorem 1: Input-to-State Stable MPC, from [13] Let Assumption 4 hold. Assume that a nominal control law $u=K(\rho) x$ exists. Consider that the MPC is given by Eq. (6), with a terminal state set given by $\mathbf{X}_{f}(\rho)$ and a terminal cost $V(x, \rho)$. Then, input-to-state stability is ensured if the following conditions hold $\forall \rho \in \mathcal{P}$ :

\footnotetext{
${ }^{4}$ This nominal control policy is purely fictional, used simply to demonstrate stability and recursive feasibility properties. We note, anyhow, that it stands for the infinite-horizon parameter-dependent Linear Quadratic Regulator (LQR) solution, which verifies $K(\rho)=$ $\arg \min _{K \in \mathbb{R}^{n_{x} \times n_{u}}}\left(\sum_{i=1}^{+\infty}\|x(k+i \mid k)\|_{Q}^{2}+\|K x(k+i-1)\|_{R}^{2}\right)$ and the admissibility on $x$ and $u$.
}

(C1) The origin lies in the interior of $\mathbf{X}_{f}(\rho)$;

(C2) Any consecutive state to $x$, in closed-loop given by $(A(\rho)+B(\rho) K(\rho)) x$ lies within $\mathbf{X}_{f}(\rho)$;

(C3) The discrete algebraic Ricatti equation is verified within this invariant set, this is, $\forall x \in \mathbf{X}_{f}(\rho)$ and $\forall \rho \in \mathcal{P}$ and $\forall \partial \rho \in \partial \mathcal{P}: V((A(\rho+B(\rho) K(\rho)) x, \rho+\partial \rho)-V(x, \rho) \leq$ $-x^{T} Q x-x^{T}\left(K(\rho)^{T} R K(\rho) x\right.$.

(C4) The image of the nominal feedback lies within the admissible control domain: $K(\rho) x \in \mathcal{U}, \forall \rho \in \mathcal{P}$.

(C5) The terminal set $\mathbf{X}_{f}(\rho)$ is a subset of $\mathcal{X}$.

Assuming that the initial solution of the MPC problem $U_{k}^{\star}$, computed with respect to an initial state $x(0)$, is feasible, then, the MPC algorithm is recursively feasible, asymptotically stabilizing the state origin.

Proof: This is a standard Theorem, whose proof is found by ensuring a energy-dissipative decay of the MPC cost function, see e.g. [13].

Theorem 2: Terminal Ingredients, adapted from [12]

The conditions (C1)-(C5) of Theorem 1 are satisfied if there exist a symmetric parameter-dependent positive definite matrix $P(\rho): \mathbb{R}^{n_{p}} \rightarrow \mathbb{R}^{n_{x} \times n_{x}}$, a parameter-dependent rectangular matrix $W(\rho): \mathbb{R}^{n_{p}} \rightarrow \mathbb{R}^{n_{u} \times n_{x}}$ and a scalar $0<\hat{\alpha}_{P} \in \mathbb{R}$ such that $Y(\rho)=(P(\rho))^{-1}>0$, $W(\rho)=K(\rho) Y(\rho)$ and that LMIs (10)-(11) hold for all $\rho \in \mathcal{P}$ and $\partial \rho \in \partial \mathcal{P}$, while minimizing $\hat{\alpha}_{P}$, where $I_{j}$ denotes the $j$-th row of the corresponding identity matrix $\mathbb{I}$.

Proof: We proceed by demonstrating that the resulting $P(\rho)$ satisfies all five conditions of Theorem 1. (C1) trivially holds due to the ellipsoidal form of $\mathbf{X}_{f}$. (C2) is verified due to the fact that $\mathbf{X}_{f}$ is a sub-level set of the terminal cost $V(\cdot)$. Therefore, if condition (C3) is verified, (C2) is consequently ensured.

The discrete Ricatti condition (C3) is verified through the solution of LMI (10). Since $Q^{-1}>0, R^{-1}>0$ and $Y(\rho+$ $\partial \rho)>0$, we can take $W(\rho)=K(\rho) Y(\rho)$ and apply two consecutive Schur, complements. This procedures leads to:

$$
\begin{gathered}
\left(Y(\rho)(A(\rho)+B(\rho) K(\rho))^{T}\right)(Y(\rho+\partial \rho))^{-1} \\
(A(\rho)+B(\rho) K(\rho)) Y(\rho)-Y(\rho) \leq \\
-Y(\rho) Q Y(\rho)-Y(\rho)(K(\rho))^{T} R K(\rho) Y(\rho)
\end{gathered} .
$$

This condition can be pre and post-multiplied by $x^{T} P(\rho)$ and $P(\rho) x$, respectively, which leads to:

$$
\begin{gathered}
x^{T}(A(\rho)+B(\rho) K(\rho))^{T} P(\rho+\partial \rho)(A(\rho)+B(\rho) K(\rho)) x \\
-x^{T} P(\rho) x \leq-x^{T} Q x-x^{T}(K(\rho))^{T} R K(\rho) x .
\end{gathered}
$$

This inequality is a sufficient condition for (C3) with $V(\cdot)$ in the form of Eq. (9).

The fourth and fifth conditions (C4-C5) are verified by the direct application of the Schur complement to Eq. (11a) and Eq. (11b), respectively, using $W(\rho)=K(\rho) Y(\rho)$. They lead, respectively, to:

$$
\begin{aligned}
\left(I_{i} K(\rho)\right)\left(\alpha_{P} Y(\rho)\right)\left(I_{i} K(\rho)\right)^{T} & \leq \bar{u}_{i}^{2} . \\
I_{j}^{T}\left(\alpha_{P} Y(\rho)\right) I_{j} & \leq \bar{x}_{i}^{2} .
\end{aligned}
$$


Since the maximum normed $F x$ of an $x$ that belongs to some ellipsoid $x^{T} P x \leq \alpha$ is given by $\sqrt{F^{T}\left(\alpha P^{-1}\right) F}$, it holds that the first inequality implies that the projection $I_{i} K(\rho) x$ (i.e. $i$-th control signal) is upper-bounded, in norm, by $\bar{u}_{i}$, which satisfies (C4). Analogously, the second inequality ensures that the projection $I_{j} x$ (i.e. $j$-th state) is norm-bounded by $\bar{x}_{j}$, which satisfies condition (C5). This concludes the proof.

We note that the above proof demonstrates that the solution of the LMIs presented in Theorem 2 ensure a positive definite parameter dependent matrix $P(\rho)$ which can be used to compute the MPC terminal ingredients $V(\cdot)$ and $\mathbf{X}_{f}$ such that input-to-state stability of the closed-loop in guaranteed, verifying the conditions of Theorem 1. Furthermore, when the MPC is designed with these terminal ingredients, for whichever initial condition $x(0) \in \mathbf{X}_{f}$ it starts with, it remains recursively feasible for all consecutive discrete time instants $k>0$.

Theorem 2 provides infinite-dimensional LMIs, that must hold $\forall \rho \in \mathcal{P}$ and $\forall \partial \rho \in \partial \mathcal{P}$, which appear due to the time-variant condition (C3). In practice, this issue is addressed by enforcing the LMIs over a sufficiently dense grid of points $(\rho, \partial \rho)$ along the $\mathcal{P} \times \partial \mathcal{P}$ plane. This converts the problem into an $n_{g}$-dimensional LMI problem, being $n_{g}$ the number of gridding points. The continuity of matrices $A(\rho)$ and $B(\rho)$ should be verified along the grid. The parameter-dependency of $P$ may be dropped if the system is quadratically stabilizable, but this may result in quite conservative performances.

$$
\begin{aligned}
& {\left[\begin{array}{cc|cc}
Y(\rho) & \star & \star & \star \\
(A(\rho) Y(\rho)+B(\rho) W(\rho)) & Y(\rho+\partial \rho) & \star & \star \\
\hline Y(\rho) & 0 & Q^{-1} & \star \\
W(\rho) & 0 & 0 & R^{-1}
\end{array}\right] \geq 0,} \\
& \text { (a) }\left[\begin{array}{c|c}
\hat{\alpha}_{P} \bar{u}_{i}^{2} & I_{i} W(\rho) \\
\hline \star & Y(\rho)
\end{array}\right] \geq 0, i \in \mathbb{N}_{\left[1, n_{u}\right]} \text {, (b) }\left[\begin{array}{c|c}
\hat{\alpha}_{P} \bar{x}_{j}^{2} & I_{j} Y(\rho) \\
\hline I_{j}^{T} & \mathbb{I}_{n_{x}}
\end{array}\right] \geq 0, j \in \mathbb{N}_{\left[1, n_{x}\right]} \text {. }
\end{aligned}
$$

\section{Semi-Active Suspension Application}

In order to validate the proposed short-sighted control method, we consider the vertical dynamics of a $1 / 5$-scaled vehicle equipped with 4 SA dampers ${ }^{5}$ and nonlinear springs. These dynamics are found through Newton's second law of motion:

$$
\begin{aligned}
m_{s} \ddot{z}_{s}(t) & =-F_{s}(t)-F_{d}(t), \\
m_{u s} \ddot{z}_{u s}(t) & =F_{s}(t)+F_{d}(t) \underbrace{-k_{t}\left(z_{u s}(t)-w(t)\right)}_{\text {Tire force }},
\end{aligned}
$$

which comprise the displacements of chassis $z_{s}(t)$ and of the wheel $z_{u s}(t)$, due to the road disturbances $w(t)$. The control input to the system is a voltage source which provides an electrical field that controls the viscosity of the electrorheological damping fluid. In practice, $u$ is simply the duty cycle of a PWM signal that controls the damping force. The nonlinear spring force is given according [14], i.e.:

$$
F_{s}(t)=k_{s_{1}}\left(z_{s}(t)-z_{u s}(t)\right)+k_{s_{2}}\left(z_{s}(t)-z_{u s}(t)\right)^{3},
$$

while the dynamic SA damping force [15] is given by:

$$
\begin{array}{r}
F_{d}(t)=k_{0}\left(z_{s}(t)-z_{u s}(t)\right)+c_{0}\left(\dot{z}_{s}(t)-\dot{z}_{u s}(t)\right)+F_{E R}(t), \\
\tau \dot{F}_{E R}(t)+F_{E R}(t)= \\
f_{c} \tanh \left(k_{1}\left(z_{s}(t)-z_{u s}(t)\right)+c_{1}\left(\dot{z}_{s}(t)-\dot{z}_{u s}(t)\right)\right) u(t) .
\end{array}
$$

Taking the system states as $x(k)=$ $\left[\begin{array}{lllll}z_{s}(k) & \dot{z}_{s}(k) & z_{u s}(k) & \dot{z}_{u s}(k) & F_{E R}(k)\end{array}\right]^{T}$

${ }^{5}$ Refer to http://www.gipsa-lab.fr/projet/inove/. All results are shown considering the front-left corner of the vehicle; similar results were obtained for the other corners. corresponding qLPV model $^{6}$ on the form of Eq. (1) is found, with two scheduling parameters $\rho=\left\{\rho_{1}, \rho_{2}\right\}$, being $\rho_{1}=\left(z_{s}(t)-z_{u s}(t)\right)^{2}$ and $\rho_{2}=\tanh \left(k_{d_{1}}\left(z_{s}(t)-z_{u s}(t)\right)+c_{2}\left(\dot{z}_{s}(t)-\dot{z}_{u s}(t)\right)\right)$, and using an Euler discretization method with a sampling period of $T_{s}=5 \mathrm{~ms}$ (operational constraint of this vehicle system). The model parameters are: $m_{s}=2.27 \mathrm{~kg}, m_{u s}=0.32 \mathrm{~kg}$, $k_{t}=12270 \mathrm{~N} / \mathrm{m}, k_{s_{1}}=1396 \mathrm{~N} / \mathrm{m}, k_{s_{2}}=0.510^{8} \mathrm{~N} / \mathrm{m}^{3}$, $k_{0}=170.4 \mathrm{~N} / \mathrm{m}, c_{0}=68.83 \mathrm{~N} / \mathrm{ms}, k_{1}=218.16 \mathrm{~N} / \mathrm{m}$, $c_{1}=21 \mathrm{~N} / \mathrm{ms}, f_{c}=28.07 \mathrm{~N}, \tau=43 \mathrm{~ms}$.

The regulation goal for this vehicle system is to minimize both chassis and wheel accelerations $\left(\ddot{z}_{s}\right.$ and $\left.\ddot{z}_{u s}\right)$, seeking a smoother and more comfortable drive for the passengers together with safety concerns (better road holding) [3]. This goal is expressed through the performance cost $J_{\mathrm{C}}=\sum_{j=0}^{+\infty}\left(a_{s} \ddot{z}_{s}^{2}(k+j \mid k)+a_{u s} \ddot{z}_{u s}^{2}(k+j \mid k)\right)$, being $a_{1}$ and $a_{2}$ unitary trade-off weights. In order to further enhance comfort, they are taken, taken, respectively, as 0.95 and 0.05 . From an MPC perspective, this cost is translated as a finitehorizon quadratic function in the form of $\ell(\cdot)$ in Eq. (5), where $Q$ and $R$ are those from [4]. W.r.t. the admissibility ${ }^{7}$ of this regulation, Assumptions 1 and 2 must be respected; the constraints of this system, the scheduling set $\mathcal{P}$ and the scheduling variation rate set $\partial \mathcal{P}$ are presented in Table I.

Concerning this application, we present realistic numeric

\footnotetext{
${ }^{6}$ Matrices can be found in [15], adjusting the spring force term in order to include the nonlinearity $k_{s_{2}}\left(z_{s}(t)-z_{u s}(t)\right)^{3}$ from [14].

${ }^{7}$ The input admissibility is given as $u \in[0,0.35]$. Therefore, in order to use the input trajectory feasibility set in the form of Eq. (4), $u$ is replaced by $u_{c}+0.175$, where $u_{c}$ is the control input used for synthesis, which is constrained according to $\left\|u_{c}\right\|^{2} \leq \sqrt{0.175}$.
} 
TABLE I

STATE AND INPUT CONSTRAINTS

\begin{tabular}{|c|c|}
\hline Var. Description & Constraint \\
\hline Chasis Displacement & $\left\|x_{1}\right\|^{2} \leq \sqrt{0.125} \mathrm{~m}$ \\
\hline Chasis Velocity & $\left\|x_{2}\right\|^{2} \leq \sqrt{2.5} \mathrm{~m} / \mathrm{s}$ \\
\hline Wheel Displacement & $\left\|x_{3}\right\|^{2} \leq \sqrt{0.125} \mathrm{~m}$ \\
\hline Wheel Velocity & $\left\|x_{4}\right\|^{2} \leq \sqrt{2.5} \mathrm{~m} / \mathrm{s}$ \\
\hline Semi-Active Damping Force & $\left\|x_{5}\right\|^{2} \leq \sqrt{10} \mathrm{~N}$ \\
\hline Control Input & $0 \leq u \leq 0.35$ \\
\hline Spring Nonlinearity & $\rho_{1} \in[0,20] 10^{-6} \mathrm{~m}$ \\
" Variation Rate & $\partial \rho_{1} \in[-2.5,2.5] 10^{-6} \mathrm{~m}$ \\
\hline $\begin{array}{c}\text { Input Nonlinearity } \\
\text { "Variation Rate }\end{array}$ & $\rho_{2} \in[-1,1]$ \\
& $\partial \rho_{2} \in[-0.5,0.5] 10^{-3} \mathrm{~m}$ \\
\hline
\end{tabular}

simulation results obtained ${ }^{8}$ with the aid of softwares packages Matlab, Yalmip and fmincon (NP) and Gurobi (QP) solvers. The system is emulated with a high-fidelity $7 \mathrm{DOF}$ validated nonlinear model [3]. Two different experiments are conducted: (A) firstly, we tune the MPC prediction horizon according to the proposed short-sighted heuristics and (B) we compare the performances achieved with the proposed method against those with other approaches from the literature. For both these scenarios, Theorem 2 is solved under a sufficiently dense grid of $(\rho, \partial \rho)$ points.

\section{A. Tuning of the Prediction Horizon}

In order to heuristically tune the size of the short-sighted prediction horizon (given in number of discrete-time samples), the considered suspension system is tested with a frequency-rich $5 \mathrm{~mm}$ chirp road profile $w$ (ISO 8608 type $\mathrm{C}$ ), which implies in a great deal of vertical trepidation, i.e. $w(t)=0.005 \sin \left(t^{2.5}\right)$. The MPC tries to counter-act the influences of $w$ upon the vertical acceleration variables, according to $J_{\mathrm{C}}$. W.r.t. the proposed heuristic criterion in Eq. (7), Table II shows the different $N_{p}$-dependent computational stress index values $t_{c}=t_{c}^{\mathrm{Min}}+t_{c}^{\mathrm{Max}}$ (i.e. respective to the min. QP and to the max. NP), compared with the NRMS ${ }^{9}$ index of the performance cost. As it can be seen, the performances increase (smaller RMS) with larger horizons, but so does the average computational time necessary to implement the strategy. We note that this is a Pareto optimality issue, since better performances are provided with longer horizons, whereas the optimization becomes more demanding as they increase. Fig. 1 illustrates two Pareto planes, showing how the NRMS index of $\ddot{z}_{s}$ (comfort) and $\ddot{z}_{u s}$ (safety) grow smaller as $N_{p}$ increases, while the computational stress $t_{c}$ increases. Therefore, w.r.t. the previous discussions, we proceed with a short-sighted horizon of $N_{p}=6$ steps for this SA suspension application, which enables its real-time operation under the threshold of $T_{s}=5 \mathrm{~ms}$.

\section{B. Performance Evaluation}

Considering the application of the short-sighted strategy with a horizon of $N_{p}=6$ steps, we proceed by compar-

\footnotetext{
${ }^{8}$ In an 15 CPU@2.4 GHz (2 Cores) Macintosh with 8 GB of RAM.

${ }^{9}$ The normalization is given w.r.t. to the passive damper condition.
}

TABLE II

Scenario (A): Prediction Horizon Tuning Sets

\begin{tabular}{|c|c|cc|c|}
\hline$N_{p}$ & NRMS $\left\{J_{\mathrm{C}}\right\}$ & $t_{c}^{\text {Min. }}$ & $t_{c}^{\text {Max. }}$ & $t_{c}$ \\
\hline 2 & 0.4132 & $0.44 \mathrm{~ms}$ & $2.51 \mathrm{~ms}$ & $2.95 \mathrm{~ms}$ \\
3 & 0.3240 & $0.46 \mathrm{~ms}$ & $3.45 \mathrm{~ms}$ & $4.01 \mathrm{~ms}$ \\
4 & 0.3234 & $0.48 \mathrm{~ms}$ & $3.56 \mathrm{~ms}$ & $4.05 \mathrm{~ms}$ \\
5 & 0.3221 & $0.49 \mathrm{~ms}$ & $3.79 \mathrm{~ms}$ & $4.29 \mathrm{~ms}$ \\
\hline 6 & 0.3220 & $0.54 \mathrm{~ms}$ & $4.17 \mathrm{~ms}$ & $4.72 \mathrm{~ms}$ \\
\hline 7 & 0.3203 & $0.55 \mathrm{~ms}$ & $4.47 \mathrm{~ms}$ & $5.02 \mathrm{~ms}$ \\
\hline
\end{tabular}
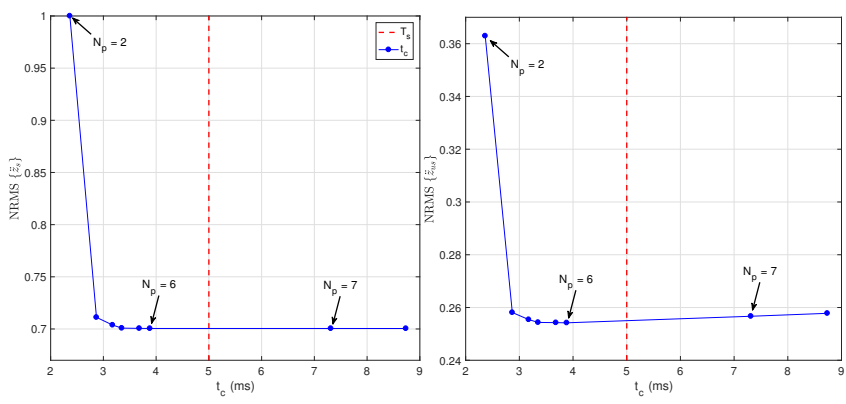

Fig. 1. Scenario (A): Pareto Planes $\ddot{z}_{s} \times t_{c}$ and $\ddot{z}_{u s} \times t_{c}$

ing our method (denoted SSMPC) against others from the literature: a) a full-blown NMPC method, which solves a nonlinear program by embedding the scheduling functions as nonlinear predictions, denoted NMPC; and b) a sub-optimal LPV MPC method [4], which uses a frozen guess for the scheduling parameters along the horizon, denoted SOMPC. Both these methods are implemented with the same cost function $\ell(\cdot)$, i.e. Eq. (5) without the terminal cost. They are designed with a horizon of $N_{p}=25$ steps, as done in recent SA suspensions MPC applications [4]. "Passive" denotes a passive suspensions system, i.e. $u=0$.

The following experiment is tested: the car is running in a straight line on a dry road, when it encounters a sequence of $5 \mathrm{~mm}$ bumps on all its wheels, exciting a bouncing motion. The obtained performances are illustrated through $\ddot{z}_{s}$, shown in Fig. 2. The corresponding control and scheduling variables are shown in Fig. 3.

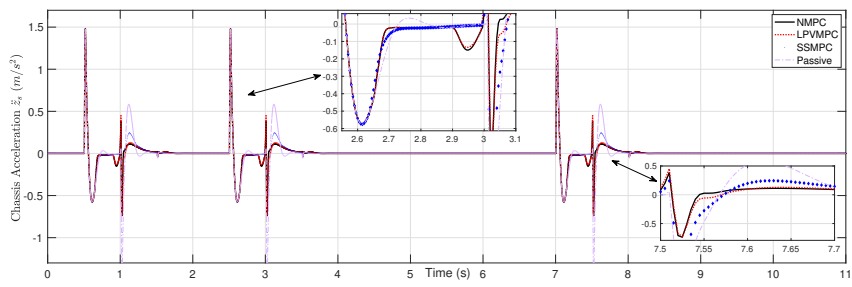

Fig. 2. Scenario (B): Time-Domain Performances $\ddot{z}_{s}$

A quantitative assessment of the achieved results is presented in Table III, which compares the RMS index of 


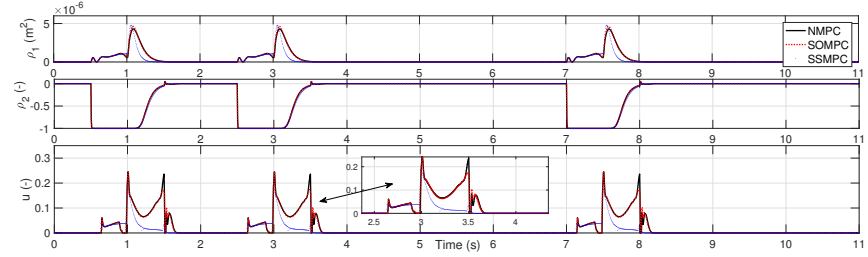

Fig. 3. Scenario (B): Control Input and Scheduling Variables

the performance cost, the Total Variance (TV) index ${ }^{10}$ of the control signal, and the average computational time $t_{c}$ achieved with each strategy. Clearly, the best performances would be obtained with the NMPC method (as expected), but it is not able to be implemented in real-time (since $t_{c}>T_{s}$ ), which is expected due to the use of nonlinear predictions. Anyhow, the SOMPC and the SSMPC methods yield comparable performances to those of the NMPC, while the proposed framework provides a smoother control signal (smaller TV index). As seen in Fig. 2, the proposed method is able to perform comparably to the other strategies, with equivalent vertical accelerations, providing a comfortable ride to passengers.

TABLE III

Scenario (B): Performance Results

\begin{tabular}{|c|c|c|c|c|c|}
\hline Method & $\operatorname{RMS}\left\{\ddot{z}_{u s}\right\}$ & $\operatorname{RMS}\left\{\ddot{z}_{u s}\right\}$ & $\operatorname{RMS}\left\{J_{\mathrm{C}}\right\}$ & $\mathrm{TV}$ & $t_{c}$ \\
\hline NMPC & 0.1168 & 0.9199 & 0.4326 & 3.73 & $703 \mathrm{~ms}$ \\
\hline SSMPC & 0.1187 & 0.9236 & 0.4341 & 1.41 & $3.13 \mathrm{~ms}$ \\
\hline SOMPC & 0.1179 & 0.9222 & 0.4336 & 3.37 & $1.96 \mathrm{~ms}$ \\
\hline Passive & 0.1553 & 0.9284 & 0.4723 & - & - \\
\hline
\end{tabular}

Finally, in order to experimentally demonstrate the validity of Theorem 2, Fig. 4 shows two 2D cuts of the control invariant ellipsoid $\mathcal{X}_{f}(\rho)$, for different instances of $\rho$ ) and the corresponding system trajectories. Clearly, the proposed SSMPC method ensures input-to-state feasibility and recursive feasibility with initial conditions given inside this set. We note that this property is not theoretically verified for the SOMPC method.
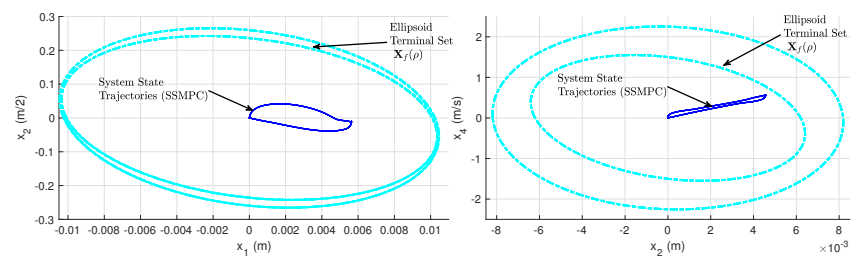

Fig. 4. Scenario (B): Domain of Attraction and Plane Trajectories

\footnotetext{
${ }^{10}$ This index measures the total variation of the control signal over the simulation, this is: $\mathrm{TV}=\sum_{k=0}^{\infty}|\delta u(k)|=\sum_{k=0}^{\infty}|u(k+1)-u(k)|$. Bigger values for TV indicate that more variation is applied to the control along the simulation. Values closer to zero indicate better (smoother) control strategies in terms of the use of the actuator.
}

\section{CONCLUSIONS}

This paper elaborated on a novel LPV MPC algorithm for semi-active suspensions systems, using a short-sighted paradigm, with small prediction horizons. The method solves a robust min-max procedure online, given w.r.t. to the bounds on the variation rates of the scheduling parameters. The strategy ensures input-to-state stability of the closed-loop with terminal ingredients, computed offline via LMIs. The algorithm achieves good performances, comparable to other techniques from the literature. The method is an elegant alternative to the available full-blown robust NMPCs and to the sub-optimal LPV MPC procedures, with intermediate computational complexity, while theoretically maintaining optimality and stability.

\section{REFERENCES}

[1] C. Poussot-Vassal, C. Spelta, O. Sename, S. M. Savaresi, and L. Dugard, "Survey and performance evaluation on some automotive semi-active suspension control methods: A comparative study on a single-corner model," Annual Reviews in Control, vol. 36, no. 1, pp. $148-160,2012$.

[2] C. E. Beal and J. C. Gerdes, "Model predictive control for vehicle stabilization at the limits of handling," IEEE Transactions on Control Systems Technology, vol. 21, no. 4, pp. 1258-1269, 2013.

[3] M. M. Morato, M. Q. Nguyen, O. Sename, and L. Dugard, "Design of a fast real-time LPV model predictive control system for semi-active suspension control of a full vehicle," Journal of the Franklin Institute, 2018.

[4] M. M. Morato, J. E. Normey-Rico, and O. Sename, "A Sub-Optimal Recursively Feasible Linear Parameter Varying Predictive Algorithm for Semi-Active Suspension Control," 2020, preprint, Submitted to IET Control Theory \& Applications. [Online]. Available: https://hal.archives-ouvertes.fr/hal-02924234

[5] E. F. Camacho and C. Bordons, Model predictive control. Springer Science \& Business Media, 2013.

[6] H. S. Abbas, R. Toth, M. Petreczky, N. Meskin, and J. Mohammadpour, "Embedding of nonlinear systems in a linear parameter-varying representation," IFAC Proceedings Volumes, vol. 47, no. 3, pp. 69076913, 2014.

[7] C. Hoffmann and H. Werner, "A survey of linear parameter-varying control applications validated by experiments or high-fidelity simulations," IEEE Transactions on Control Systems Technology, vol. 23, no. 2, pp. 416-433, 2014.

[8] M. M. Morato, J. E. Normey-Rico, and O. Sename, "Model predictive control design for linear parameter varying systems: A survey," Annиal Reviews in Control, vol. 49, pp. 64 - 80, 2020.

[9] H. S. Abbas, J. Hanema, R. Tóth, J. Mohammadpour, and N. Meskin, "A new approach to robust MPC design for LPV systems in inputoutput form," IFAC-PapersOnLine, vol. 51, no. 26, pp. 112-117, 2018

[10] P. S. Cisneros, S. Voss, and H. Werner, "Efficient nonlinear model predictive control via quasi-LPV representation," in IEEE Conference on Decision and Control. IEEE, 2016, pp. 3216-3221.

[11] M. M. Morato, J. E. Normey-Rico, and O. Sename, "Novel qLPV MPC design with least-squares scheduling prediction," IFACPapersOnLine, vol. 52, no. 28, pp. 158-163, 2019.

[12] P. S. González Cisneros and H. Werner, "Nonlinear model predictive control for models in quasi-linear parameter varying form," International Journal of Robust and Nonlinear Control, vol. n/a, no. n/a, 2020.

[13] D. Q. Mayne, J. B. Rawlings, C. V. Rao, and P. O. Scokaert, "Constrained model predictive control: Stability and optimality," $\mathrm{Au}$ tomatica, vol. 36, no. 6, pp. 789-814, 2000.

[14] W. Sun, H. Gao, and O. Kaynak, "Vibration isolation for active suspensions with performance constraints and actuator saturation," IEEE/ASME transactions on mechatronics, vol. 20, no. 2, pp. 675683, 2014.

[15] T.-P. Pham, O. Sename, and L. Dugard, "Real-time damper force estimation of vehicle electrorheological suspension: A nonlinear parameter varying approach," IFAC-PapersOnLine, vol. 52, no. 28, pp. 94-99, 2019. 